\title{
Investigating the effects of reduced technological constraints on cycle time through simulation modelling for automated steel wall framing
}

\author{
Nabeel MALIK ${ }^{1}$, Rafiq AHMAD ${ }^{2 *}$ and Mohamed AL-HUSSEIN ${ }^{3}$ \\ ${ }^{1}$ MSc Student, Department of Civil and Environmental Engineering, University of Alberta \\ ${ }^{2}$ Professor, Department of Mechanical Engineering, University of Alberta \\ ${ }^{3}$ Professor, Department of Civil and Environmental Engineering, University of Alberta \\ "Corresponding author's e-mail: rafiq.ahmad@ualberta.ca
}

\begin{abstract}
Off-site construction constitutes a paradigm shift in construction promoting improved sustainability. At present, North America's building construction sector is still dominated by conventional stick-built construction, which is prone to excessive material waste, longer cycle times, high labour costs, and lower quality. In contrast, inspired by the manufacturing industry, off-site construction is an approach in which building components are prefabricated in factories and transported to the construction site for on-site assembly. As the concept of off-site prefabrication gains momentum within the domain of construction, some home builders are bringing the traditional industry practice into a factory setting, thus resulting in stick-buildingunder-a-roof. This paper describes the development of simulation models for the automated light gauge steel framing process using discrete-event simulation mimicking real-time machine production capacity and cycle time. At present, the literature on the development of such models for automated construction machinery is lacking; in this context, this paper aims to showcase the advantages of simulation as a decision-making support tool. Construction of such models provides a useful tool for understanding bottlenecks in machine operations that can be addressed to meet local demands. Since the steel framing process primarily consists of manual assembly and fastening of cold-formed steel (CFS) frames, these models showcase the potential to increase the level of automation through the addition of various mechanical and control modifications to an existing prototype steel framing machine. The results show that cycle time reductions of 13 percent or greater are possible by applying the proposed modifications.
\end{abstract}

\section{KEYWORDS}

Offsite construction, steel framing, automation, simulation, cycle times

\section{INTRODUCTION}

Cold-formed steel (CFS) frames are commonly used for constructing interior and exterior wall frames for mid-size residential and commercial projects, and in fact in many jurisdictions building codes require the use of CFS in structures taller than 6-storeys. However, this method of construction usually requires excessive manual work including handling of heavy steel pieces. In addition to safety and ergonomic issues, these manual operations also result in lower productivity, 
reduced quality of work, and increased material waste. These challenges associated with traditional construction can be circumvented by adopting modern methods of construction that promote tangible benefits through pre-fabricated construction (Lawson and Ogden 2008).

Prefabricated construction provides a cost-effective solution, with most of the building components produced off-site in a controlled factory environment. Paudel et al. (2016) show that the use of prefabrication and preassembly has increased by $86 \%$ over the past 15 years. They also pointed out that prefabricated construction provides significant cost and time savings. A case study (Shahzad et al. 2015) compares 66 building projects and finds that prefabrication on an average leads to a $34 \%$ reduction in completion time and a $19 \%$ reduction in completion costs. Through increased automation and innovation, such advantages can further the adoption of modern methods of construction (Bock 2015).

Increased demand for higher product variability and shorter cycle times within the manufacturing industry has paved the way for extensive research around dynamic decision-making support systems for existing products (Ahmad et al. 2016; Ahmad and Plapper 2015), and increased application of Design for Excellence (DFX) methods for product design. Design for manufacturing (DFM) and Design for production (DFP) are two well-studied methodologies of DFX that involve cycle time analysis, where DFM aims to ensure the manufacturability of a component as per supplier's capability, while DFP involves evaluating manufacturing capacity and manufacturing time (Herrmann and Chincholkar 2000). As shown by Herrmann (2003) DFP and DFM techniques can improve manufacturing systems such as production lines, factories, and supply chains, where these techniques function as decision-making tools for further system enhancements. However, application of such systems in the construction industry has been lacking.

Nevertheless, advances in off-site construction are promoting greater use of simulation modelling. In particular, Discrete-event simulation (DES) is a computer-based simulation approach wherein real-world systems are converted to discrete events mimicking real-world processes. Many studies have been developed that apply DES to improve the production line for modular construction (Altaf et al. 2014, 2015; Liu et al. 2015). Simphony has been used extensively to construct such simulation models due to its close association with the construction domain, where it provides tools for assessment of project duration, resource utilization, and general decision making support (Afifi et al. 2016).

To date, the use of virtual simulation as a decision-making support tool during the prototype phase of automated construction machinery has been limited. Therefore, this paper aims to combine DES with the manufacturing reduction framework, MTTP, proposed by Johnson (2003) in order to understand the manufacturing capacity and manufacturing time of a steel framing machine prototype (SFMP). In short, this paper proposes a cycle time study in which various modifications to an existing light-gauge steel (LGS) wall panel fabrication machine prototype are investigated. Initially, the real-world machine logic is mapped using DES to validate the accuracy of utilizing such modelling techniques; moreover, the model is further used as a baseline to forecast the effect of proposed design changes. Each design modification aims to increase productivity through the removal of existing bottlenecks, where the results from various panel configurations can be used as a guideline informing future in-depth design modifications. 


\section{SYSTEM DESCRIPTION}

Figure 1 shows a brief overview of the main components of the steel framing machine prototype (SFMP). This machine follows a multi-phase semi-automated framing approach for constructing LGS wall-framed panels, wherein panels assembly is undertaken manually, and the panel fastening is automized. More specifically, the process starts with uploading of manufacturing plans via a human-machine interface (HMI), which allows the machine to adjust its dimensions to allow for the panel assembly. The process of manual assembly is undertaken on Table A, with the assembly time approximated using the panel assembly equation (1) from Liu et al. (2015):

$$
T_{A}=\sum_{i=1}^{N_{S T}} T_{S T}+T_{B P} N_{B P}+T_{T P} N_{T P}+T_{w} N_{w}+T_{D} T_{D}+V
$$

where $\mathrm{T}_{\mathrm{A}}=$ total assembly time; $\mathrm{T}_{\mathrm{BP}}=$ time to place bottom plate; $\mathrm{T}_{\mathrm{TP}}=$ time to place top plate; $\mathrm{T}_{\mathrm{ST}}=$ time to place a single stud; $\mathrm{T}_{\mathrm{W}}=$ time to assemble window; $\mathrm{T}_{\mathrm{D}}=$ time to assemble door; $\mathrm{V}$ $=$ variation in assembly process; and $\mathrm{N}_{\mathrm{BP}}, \mathrm{N}_{\mathrm{TP}}, \mathrm{N}_{\mathrm{ST}}, \mathrm{N}_{\mathrm{W}}$, and $\mathrm{N}_{\mathrm{D}}=$ number of subcomponents relating to the aforementioned variables.

The completion of the manual assembly phase is followed by an automatic screw-fastening phase, where the soft-connected panel is squared and dragged via electromagnetic squares arranged in a rectangular pattern along the corners of the assembled panel. Synchronized dragging allows the frame to be precisely positioned between the two horizontal beams of a stationary gantry, wherein four screw-fastening carriages operate in unison to place screws in predetermined locations. Considering that the carriages work in pairs, the movement of carriages is preplanned by a custom tool path algorithm designed to avoid collisions and to allow for rapid screwing operations. Since the carriages are located on a stationary gantry, the frame is repositioned to each location requiring screw-fastening operations. Finally, the automated phase ends when the framed wall panel arrives on Table B and is offloaded for further processing.

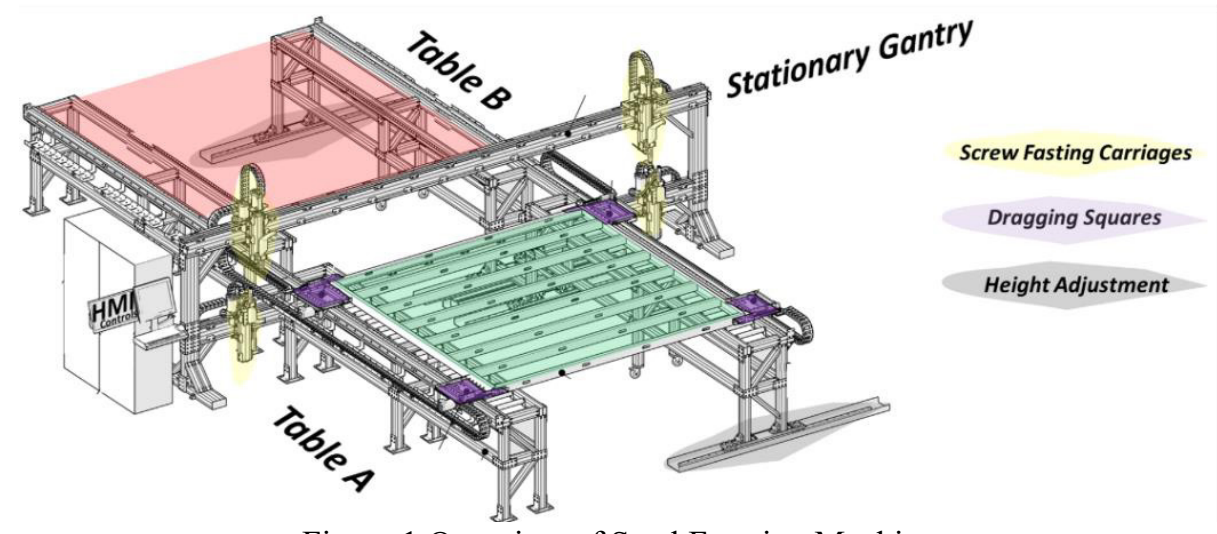

Figure 1 Overview of Steel Framing Machine.

\section{MODEL CONSTRUCTION}

Since the panel configuration varies considerably from one panel to another, the simulation model is linked to an MS Access database, which provides an accurate link between the model and the 
actual shop drawings. The following sections describe the process of utilizing the shop drawing information to construct a reliable simulation model for the production of each steel panel.

Simulation models are constructed in the general template of Simphony, using discrete-event modelling. In Simphony, each main operation is modelled using a "Task" element, which holds an entity for a specified amount of time. The data flow between different tasks is controlled using "Activator" and "Valve" elements. For any "Task" elements requiring resources, a "Capture" element is utilized to occupy available resources for the specified duration. In addition to different elements, Simphony also features local and global variables for representing attributes of entities at individual level and at simulation level, respectively. A select list of variables and their descriptions is presented in Table 1 as an example.

Table 1 Summary of select variables from simulation models (Local $=\mathrm{L}$ and Global $=\mathrm{G}$ ).

\begin{tabular}{|c|c|c|c|c|c|}
\hline Name & Description & Name & Description & Name & Description \\
\hline$\overline{\mathrm{LX}(1)}$ & $x$-position & GX(97) & Right side screw operations at given $x$ & LN(97) & Panel ID \\
\hline LX(2) & $y$-position & GX(98) & Left side screw operations at given $x$ & GX(12) & Panel ID Tracker \\
\hline LX(3) & $z$-position & GX(99) & set $x$-position after frame positioning & $\mathrm{LX}(4 / 5 / 6)$ & No. of Windows /Doors/ Studs \\
\hline
\end{tabular}

The simulation sequence begins with the creation of all the entities stored in the database. Initially, the value for GX(12) is set to 1, thereby allowing the entities corresponding to that panel ID to flow into the soft connection phase wherein a "worker" resource is acquired for the duration calculated using Eq. 1. Following this phase, the soft-connected panel is moved as per LX(1) and the said position is stored in GX(99). Here, screw-fastening operations relating to $\mathrm{GX}(99)$ are simulated using closed loops as represented by blue lines in Figure 2. The completion of screwfastening operations at a given frame position is simulated by passing two entities through an activator, which allows for the entities relating to the next frame position to pass (represented as red lines in Figure 2). This information is used to reposition the frame, after which the above process repeats until the completion of required operations. At the end of the screw-fastening phase, a short offloading delay is simulated, followed by the incrementing of GX(12) and activation of the original "valve", thereby allowing for information pertaining to the next frame to enter the simulation model (represented as green lines in Figure 2). A sample overview of the DES model is shown in Figure 3.

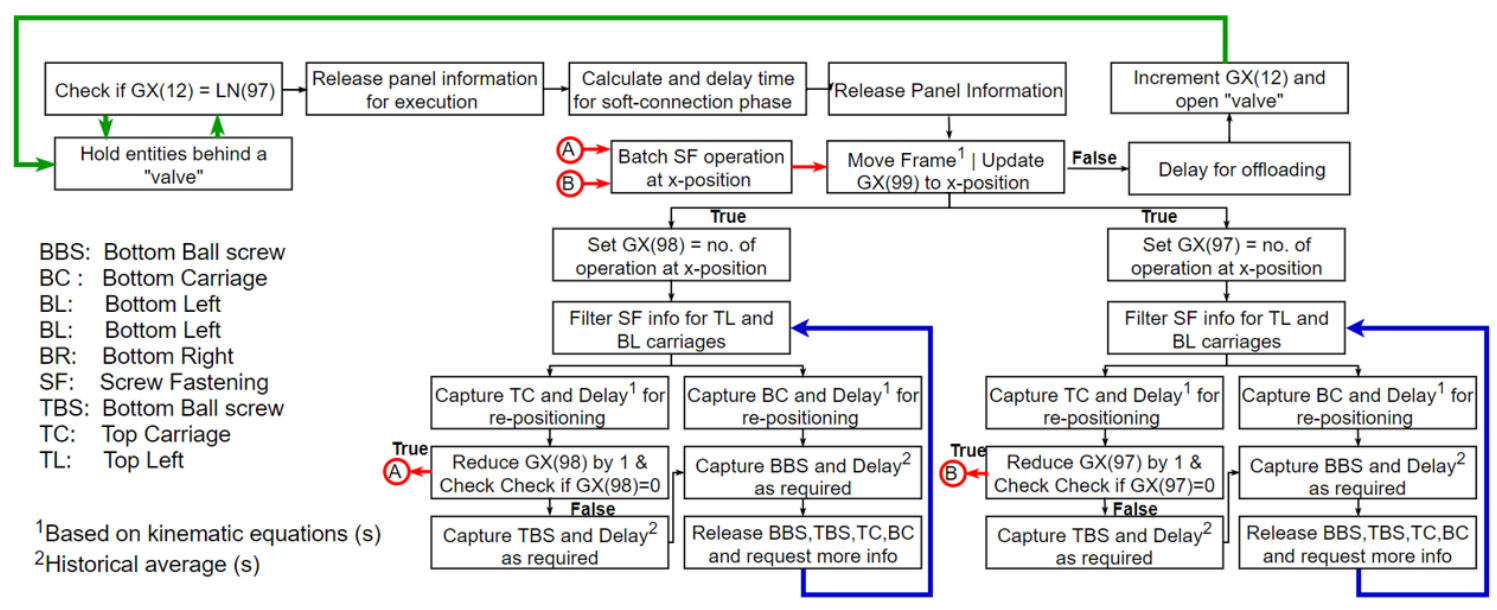

Figure 2 Main Logic for Discrete-event Simulation Model. 


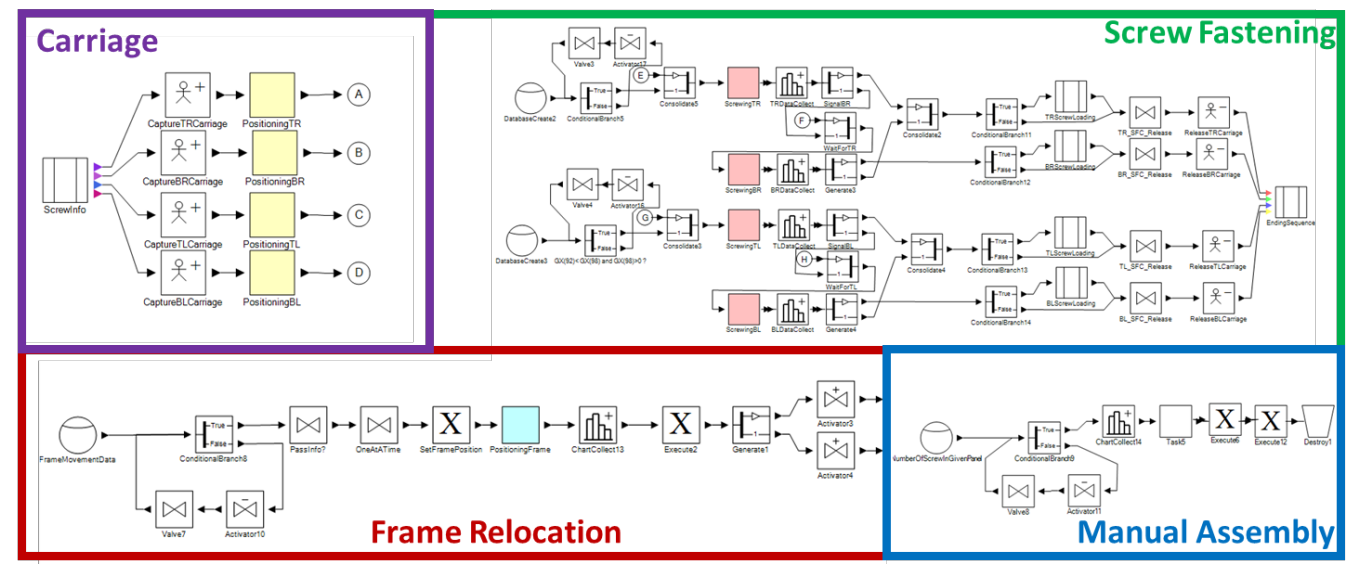

Figure 3 Simulation model of steel framing machine in Simphony.NET

\section{SCENARIO ONE: MANUAL SCREW FEEDING}

For scenario one, each screwdriver can hold only 50 screws; once the magazine of screws has been exhausted, all operations are halted for the duration of the refilling phase. As per the current prototype design, the average duration for refilling a new magazine is assumed to be 50 seconds.

\section{SCENARIO TWO: AUTO SCREW FEEDING SYSTEM}

For scenario two, the overall simulation sequence and machine logic remain the same as for scenario one. However, the screwdrivers are not limited to 50 -screw magazines. This change requires the addition of a centralized screw feeding system that allows for automatic feeding of screws through feed tubes attached to a vibrating bowl. Here, vibrating bowls have a capacity of a few hundred screws and can be refilled easily. Moreover, the length of feed tubes directly correlates to feeding rate wherein longer the tube length, the smaller the feed rate. By estimating the relationship between cycle times and screw feeding rate, one can design mechanical systems that provide the most optimal cycle times and minimize costly design changes. This scenario closely resembles actions of "reduce waiting time per part" as proposed by Johnson (2003).

\section{SCENARIO THREE: DESIGN MODIFICATION FOR FRAME CLAMPING}

For scenario three, another limitation resulting from the coupling of the top and bottom screwdrivers is investigated. Here, if the panel is not clamped from the top, the panel may be lifted by the force produced through the bottom screw-fastening operations. This high degree of interdependence, i.e. high coupling, thus requires the top screwdrivers to remain fully extended while the bottom operations are conducted, and negatively affects the cycle time during the hard connection phase. However, the addition of pneumatic clamping mechanisms can allow both screwdrivers to operate synchronously, thereby allowing for faster cycle times. This scenario closely resembles actions of "reduce processing time per part" as proposed by Johnson (2003).

\section{SCENARIO FOUR: HYBRID DESIGN WITH CLAMPING AND FEEDING}

Since the design changes as stated in scenario two and three would require the addition of a pneumatic system, scenario four examines the potential of implementing both modifications working in tandem. 


\section{MODEL INPUT DATA}

To illustrate the effects of various panel configurations on cycle times, Table 2 shows a summary of selected panels with and without building apertures. Since each of the above scenarios necessitates changing a certain aspect of the machine's logic, the variability in panel designs is crucial for evaluating the effect on cycle time.

Table 2 Summary panels utilized for modelling cycle times.

\begin{tabular}{|c|c|c|c|c|c|}
\hline Panel De & & 1 & 2 & 3 & 4 \\
\hline \multicolumn{2}{|c|}{ Shop Drawing } & & & Ш & $H=$ \\
\hline \multirow{2}{*}{\multicolumn{2}{|c|}{$\begin{array}{c}\text { Frame Dimensions }(\mathrm{mm}) \\
* \text { Width }(\mathrm{z})=92\end{array}$}} & \multirow{2}{*}{$\begin{array}{l}\text { Length }(x)=3048 \\
\text { Height }(y)=2439\end{array}$} & Length $(\mathrm{x})=3048$ & \multirow{2}{*}{$\begin{array}{l}\text { Length }(\mathrm{x})=2927 \\
\text { Height }(\mathrm{y})=2607\end{array}$} & Length $(\mathrm{x})=2927$ \\
\hline & & & Height $(y)=2439$ & & Height $(y)=2607$ \\
\hline \multirow{3}{*}{$\begin{array}{c}\text { Screw- } \\
\text { fastening } \\
\text { Operations }\end{array}$} & Left & 9 & 11 & 15 & 27 \\
\hline & Right & 9 & 25 & 15 & 27 \\
\hline & Total & 36 & 72 & 60 & 108 \\
\hline
\end{tabular}

\section{RESULTS AND DISCUSSION}

Initially, the simulation model is run with the assumption of zero time associated with the assembly and offloading phase as well as infinite screw capacity. These assumptions allow for the basic validation of the simulation model as per real-world machine logic. In other words, the four panels as described in Table 2 are executed on the real-world machine and the resulting cycle times are compared to simulated times as shown in Figure 4. Here, the simulated results are comparable to real-world results, thereby allowing for a baseline validation of the given scenarios.

Figure 5 shows the impact of four scenarios while simulating continuous production of 20 wall panels. Such production consists of simulating four panels from Table 2 five times with their respective soft-connection and offloading phases. Moreover, each simulation run is repeated 100 times with an identical seed value. Here, the repetition of simulation runs captures the probabilistic nature of soft-connection and offloading phases, whereas equal seed value allows for one-to-one comparison between the hard-connection phases of the scenarios. The total production time is found to vary from 3.1 to $2.4 \mathrm{hr}$. Since scenarios one and three require manual loading of screws, these scenarios are not affected by the feeding delays, whereas scenarios two and four reflect the negative impact of lower screw-feeding rates on the final production times. Without the inclusion of cost analysis, a comparison between each scenario is not viable. Nevertheless, by utilizing the simulation results, we can conclude that the addition of auto-feeding is only acceptable if the feeding delays are less than 6.3 seconds, whereas applying the top clamping and auto-feeding should only be adopted if the feeding delays are less than 8.8 seconds. By developing systems within the given constraints, the cycle times for SFMP can be either maintained or decreased, as shown in Figure 5. Furthermore, the addition of frame clamping with manual feeding (scenario three) can result in approximately a 13 percent drop in production times as compared to scenario one.

Finally, the reason for high production time for scenario one is shown in Figure 6, where the production of 20 panels requires 13 separate stops for the refilling phase. These excessive delays for manual loading thus result in scenario one being deemed the least desirable. Figure 7 shows an 
in-depth comparison among the various feeding rates and panel configurations, where the panels with closely positioned operations experience more significant effects of screw feeding delays.

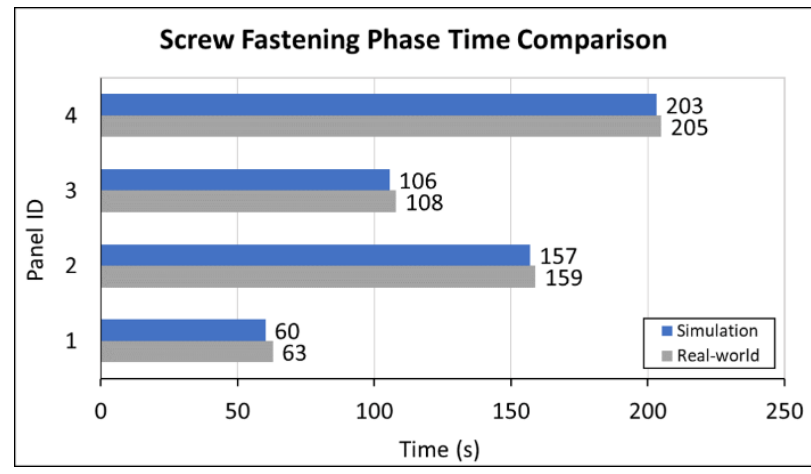

Figure 4 Screw-fastening time comparison between simulation model and real-world prototype.

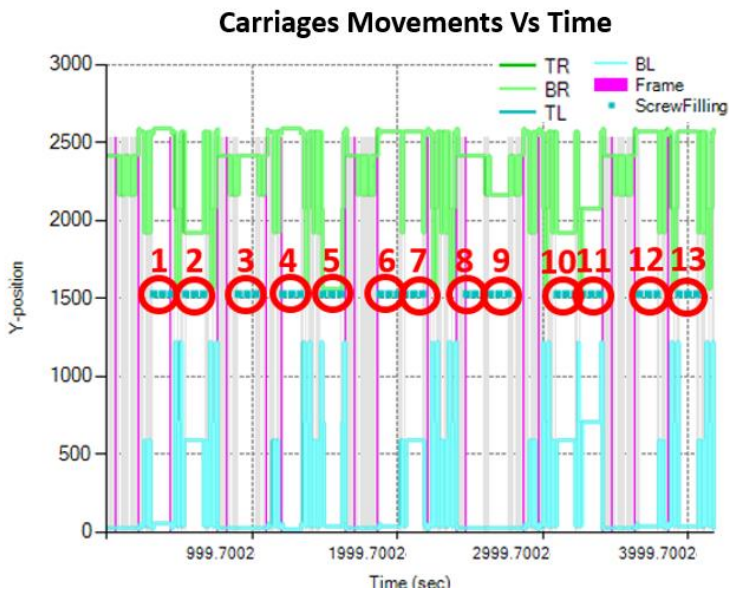

Figure 6 Carriage movements vs time comparison to produce 20 wall panels by utilizing manual screw loading (i.e. scenario 1).

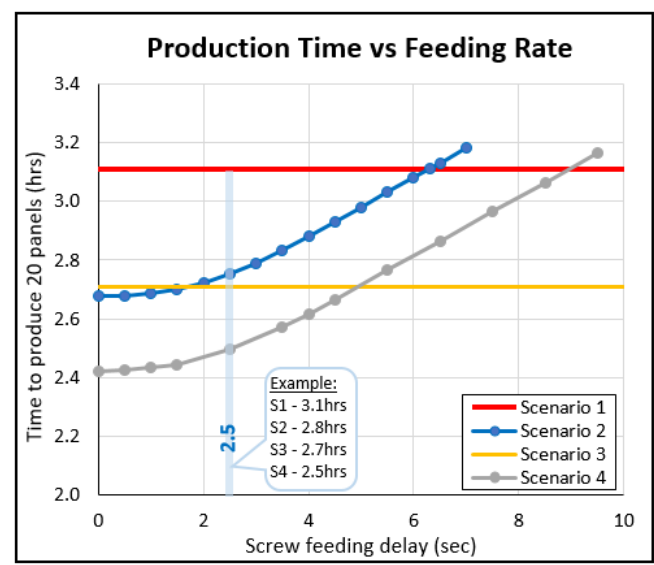

Figure 5 Comparison between time for production of 20 continuous panels and proposed scenarios.

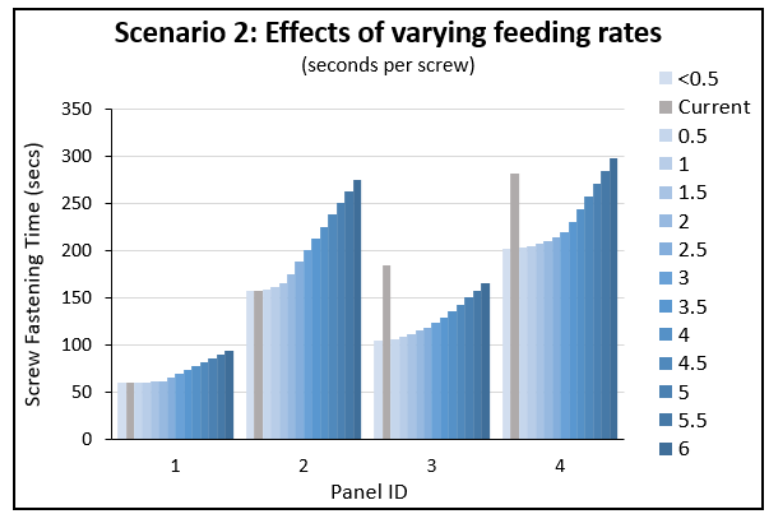

Figure 7 Understanding effects on cycle time for varying feed rates on scenario 2 .

\section{CONCLUSION}

To improve cycle times during automatic screw-fastening operations, discrete-event modelling techniques are employed for estimating the implication of various system modifications. Such improvement studies have typically been limited primarily to the manufacturing industry; therefore, the contribution of this paper is to apply throughput time reduction techniques for understanding the manufacturing capacity and manufacturing time for automated construction machinery. Through a preliminary model comparison with a real-world prototype machine, two bottlenecks relating to the coupling of screw-fastening operations and manual screw feeding are determined, while three modifications are investigated to minimize the impact of these bottlenecks. The simulation results provide valuable insights into potential modifications and their approximate effects on cycle time. For instance, for the screw fastening phase, cycle time reductions of 13 percent or greater are possible by applying proposed modifications. As a further example, by changing the screw-feeding system from manual to auto-feeding, the benefits can be immense if the screw feeding rate is kept below 6.3 seconds, whereas exceeding this threshold would result in 
higher cycle times. Detailed cost and engineering information is required to better understand the opportunity cost of each proposed solution; however, compared to manual feeding each option can be tailored to reduce cycle times.

\section{ACKNOWLEDGEMENTS}

The authors gratefully acknowledge the support of all personnel involved in the development of the steel framing machine prototype, as well as the financial support from the Natural Sciences and Engineering Research Council of Canada.

\section{REFERENCES}

Afifi, M., Al-Hussein, M., Abourizk, S., Fotouh, A., and Bouferguene, A. (2016). "Discrete and Continuous Simulation Approach to Optimize the Productivity of Modular Construction Element." ISARC. Proceedings of the International Symposium on Automation and Robotics in Construction, Vilnius Gediminas Technical University, Department of Construction Economics \& Property, 1.

Ahmad, R., and Plapper, P. (2015). "Generation of safe tool-path for 2.5 D milling/drilling machine-tool using 3D ToF sensor." CIRP Journal of Manufacturing Science and Technology, Elsevier, 10, 84-91.

Ahmad, R., Tichadou, S., and Hascoet, J.-Y. (2016). "Generation of safe and intelligent tool-paths for multi-axis machine-tools in a dynamic 2D virtual environment." International Journal of Computer Integrated Manufacturing, Taylor \& Francis, 29(9), 982-995.

Altaf, M. S., Al-Hussein, M., and Yu, H. (2014). "Wood-frame wall panel sequencing based on discrete-event simulation and particle swarm optimization." ISARC. Proceedings of the International Symposium on Automation and Robotics in Construction, Vilnius Gediminas Technical University, Department of Construction Economics \& Property, 1.

Altaf, M. S., Liu, H., Al-Hussein, M., and Yu, H. (2015). "Online simulation modeling of prefabricated wall panel production using RFID system." Winter Simulation Conference (WSC), 2015, IEEE, 3379-3390.

Bock, T. (2015). "The future of construction automation: Technological disruption and the upcoming ubiquity of robotics." Automation in Construction, 59(Supplement C), 113-121.

Herrmann, J. W. (2003). "Design for production: Concepts and applications." Proceedings of the SME East Coast Region, Citeseer, 3, 1-9.

Herrmann, J. W., and Chincholkar, M. M. (2000). "Design for production: a tool for reducing manufacturing cycle time." Proceedings of the 2000 ASME Design Engineering Technical Conference, 10-13.

Johnson, D. J. (2003). "A framework for reducing manufacturing throughput time." Journal of manufacturing systems, Society of Manufacturing Engineers (publishers), 22(4), 283.

Lawson, R. M., and Ogden, R. G. (2008). “'Hybrid' light steel panel and modular systems.” ThinWalled Structures, 46(7), 720-730.

Liu, H., Altaf, M. S., Lei, Z., Lu, M., and Al-Hussein, M. (2015). “Automated production planning in panelized construction enabled by integrating discrete-event simulation and BIM."

Paudel, P., Dulal, S., Bhandari, M., and Tomar, A. (2016). "Study on Pre-fabricated Modular and Steel Structures.” SSRG International Journal of Civil Engineering (SSRG-IJCE), 3(5), 15.

Shahzad, W., Mbachu, J., and Domingo, N. (2015). "Marginal productivity gained through prefabrication: Case studies of building projects in Auckland." Buildings, Multidisciplinary Digital Publishing Institute, 5(1), 196-208. 